asthma in adolescents and adults. The beneficial role of corticosteroid administration in reducing the need for hospitalisation in asthma episodes in adults has been demonstrated, however the evidence supporting corticosteroid use in preschool aged children with acute wheeze exacerbations is less robust.

Aim To assess the efficacy of oral prednisolone in pre-schoolaged children presenting to a paediatric emergency department (PED) with viral associated wheeze.

Methods A superiority analysis of the randomised, doubleblind placebo controlled trial, comparing the effect of a 3 day course of daily oral prednisolone $(1 \mathrm{mg} / \mathrm{kg} /$ day $)$ with placebo on the primary outcome of length of hospital stay in children (aged 24 to 72 months) presenting to a tertiary PED with VAW.

Results Between June 2012 and June 2015 we randomised 624 eligible patients with 605 (300 placebo and 305 prednisolone) being available for full analysis. The 'ready for discharge' length of stay (LOS) was significantly reduced in the prednisolone group (median [interquartile range] $370 \mathrm{~min}$ [121, 709]) compared to placebo (median [interquartile range] 540 min $(124,971))$; the unadjusted ratio of geometric means for LOS was 0.79 (95\% CI; 0.64, 0.97; $\mathrm{p}=0.0227, \mathrm{n}=605)$. The prednisolone group had significantly reduced risk, relative to placebo group, of the LOS exceeding either 7 or 12 hours, adjusted RR 0.82 (95\% CI; 0..69,0.96; p=0.0166) and 0.67 $(95 \% \mathrm{CI} ; 0 \cdot 51,0 \cdot 86 ; \mathrm{p}=0 \cdot 0018)$ respectively. Subgroup analysis demonstrated efficacy (reduced continuous LOS, and reduced risk of LOS exceeding either 7 or 12 hours) for prednisolone among patients with more severe exacerbations. The absolute reduction (difference) in the percentage of patients with a LOS exceeding 12 hours (13.7\%) represents a number needed to treat of approximately 8 patients to prevent the LOS of 1 patient exceeding 12 hours.

Conclusion Oral prednisolone demonstrated a clear benefit over placebo at reducing the LOS in children presenting to a PED with viral associated wheeze; with the greatest efficacy observed among patients with more severe features of wheeze.

\section{G331 MANIPULATION UNDER 70\% NITROUS IN A PAEDIATRIC EMERGENCY DEPARTMENT; THE EFFECTS AND CHALLENGES POST IMPLEMENTATION}

${ }^{1} \mathrm{~J}$ Le Geyt, ${ }^{2} \mathrm{~A}$ Vaughan, ${ }^{2} \mathrm{E}$ Lindisfarne, ${ }^{2} \mathrm{~T}$ Crompton, ${ }^{1} \mathrm{M}$ Lazner, ${ }^{1} \mathrm{C}$ Bevan. ${ }^{1}$ Paediatric Emergency Department, Royal Alexandra Children's Hospital, Brighton, UK; ${ }^{2}$ Orthopaedics, Royal Alexandra Children's Hospital, Brighton, UK

10.1136/archdischild-2018-rcpch.321

Aims The use of manipulation under sedation (MUS) of fractures in paediatric emergency departments (PED) has become increasingly popular. Numerous studies show MUS to be safe, effective, and reducing time to manipulation, number of MUAs (manipulation under anaesthetic) and time spent in hospital. Despite this there were many barriers and safety concerns to the introduction of MUS in our hospital. A MUS protocol using $70 \%$ Nitrous for upper limb fractures was introduced in December 2016 at our PED. The aim of our study was to evaluate this service regarding safety, cost effectiveness, barriers to its use and to evaluate admissions saved.

Methods An audit was conducted of all paediatric eligible forearm and wrist fractures requiring manipulation. Data was collected from two study periods (of 5 months) before and after implementation of protocol for comparisons, February-
July 2016 and same period in 2017. Data was collected on patient demographics, treatment modality,timings of presentation and treatments, and reasons eligible MUS were not undertaken.

Results In 2016, 36 patients who would have been eligible for MUS went on to have admissions and MUA. In 2017 there were 35 eligible patients, 20 who had MUS in the PED and 15 who had admission and MUA. Of the MUS group 100\% were successful with no complications or requirement for further surgery and were discharged home within 4 hours. Of the 15 MUA, $11(73 \%)$ the MUS was unable to occur because of staffing issues; presenting out-of-hours or the department being too busy. The average time of presentation of the MUS group was 16:42 and the MUA group 18:08, both out-ofhours for staffing levels. The MUS group waited an average 149 mins between presentation and definitive reduction, compared to 2.2 days in the MUA group.The cost saved per MUS case was average $£ 1300$.

Conclusion This study demonstrates MUS protocol in a PED is safe and cost efficient, providing a solution to the crises in inpatient beds and busy theatre lists. The biggest barrier to its regular implementation is staff availability out-of-hours. As a result of this study our department are creating a morning 'virtual list' within the ED,increasing MUS capacity within the current service.

\section{G332 ACUTE TRAUMATIC COAGULOPATHY IN THE PAEDIATRIC POPULATION: A SYSTEMATIC REVIEW}

${ }^{1} \mathrm{C}$ Lindsay, ${ }^{2} \mathrm{E}$ Broad. 'Accident and Emergency Department, Norfolk and Norwich University Hospital, Norwich, UK; ${ }^{2}$ Paedatric Department, Norfolk and Norwich University Hospital, Norwich, UK

\subsection{6/archdischild-2018-rcpch.322}

Introduction Acute traumatic coagulopathy is a clinically distinct coagulopathy resulting from the injury itself and subsequent hypoperfusion. The condition has been associated with increased morbidity and mortality in adults but little is known about the condition in children.

Objective This review aims to summarise current literature regarding the incidence of acute traumatic coagulopathy in a paediatric population and associated risk factors and outcomes.

Evidence review We carried out searches of Medline, EMBASE and Cochrane library databases as well as the following registries: clinicaltrials.gov, the European Union Clinical Trials Register and the International Standard Randomised Controlled Trial Number (ISRCTN) registry. Searches were limited to studies published from 2003, the point at which acute traumatic coagulopathy was defined. For each relevant study identified, reference and author specific searches were carried out. Finally, the grey literature was searched. Any study defining acute traumatic coagulopathy as an international normalised ratio (INR) $>1.2$, including individuals aged 17 or less with any type of traumatic injury was eligible.

Results We identified a total of nine studies that met the inclusion criteria. All studies were retrospective cohort studies deemed to be at low or uncertain risk of bias. Eight studies were conducted in trauma centres in the United States and one in combat support hospitals in Iraq and Afghanistan. Study populations ranged from 84 to 1531 participants. Acute traumatic coagulopathy was present in $31.7 \%$ (range $24.6 \%$ 40.9\%). Mortality was $29.3 \%$ (range $21.2 \%-60 \%$ ) in those 
who were coagulopathic on admission versus 3.2\% (range $0 \%-6 \%$ ) in those with normal coagulation studies. Data regarding risk factors and additional outcomes such as transfusion requirements and thrombosis were limited.

Conclusions Acute traumatic coagulopathy is present in around one third of severely injured paediatric patients and is associated with increased mortality, particularly in individuals with isolated brain injuries. The incidence of the condition in the wider paediatric trauma population who do not require admission to intensive care remains largely unknown but may be less common. There was limited data regarding the risk factors and additional outcomes associated with acute traumatic coagulopathy.

\section{G333 RAISE STUDY; A MULTICENTRED STUDY OF INFANTS PRESENTING TO UK EMERGENCY DEPARTMENTS THAT ARE INVESTIGATED FOR SKULLS FRACTURES}

J Le Geyt, L Perera, C Bevan. Paediatric Emergency Department, Royal Alexandra Children's Hospital, Brighton, UK

\subsection{6/archdischild-2018-rcpch.323}

Background Head injury in a young child is a common presentation to emergency departments. Skull fractures in young children often generate concern regarding possible non-accidental injury (NAI). Guidelines produced by the Royal College of Radiology recommend a skeletal survey and CT head in children under 2 years of age, if abusive head injury is suspected. We hypothesise that there are significant variations in practice across the UK with regards to radiological investigations performed, safeguarding referrals, assessments, and follow up.

Methods All hospitals within the PERUKI network were invited to submit data. The study population were children under 2 years who presented to the emergency department and underwent radiological investigation for a suspected skull fracture. Retrospective data was collected for presentations between January 2012 to December 2014 inclusive. Data was collected on the purported mechanisms for the injury, clinical findings, the investigative processes undertaken and subsequent follow-up.

Preliminary results 20 UK emergency departments submitted data. Data was collected for 1583 infants who underwent radiological imaging for a suspected skull fracture. 478 of these $(30 \%)$ had a confirmed skull fracture. $107(7 \%)$ had a likely or definite cause of NAI. Data analysis is ongoing; by February 2018 we will have results of variations in practice across the UK, demographics on event information, symptoms, signs, investigation results of children investigated for skull fractures, and statistical differences in the subgroups of 'no fractures found', 'fractures', and 'fractures likely caused by NAI'.

Conclusion This study will create an extensive database of children less than 2 years old presenting to emergency departments that were investigated for skull fractures. The study aims to produce evidence to inform guidance, enhance clinical decision making in determining the likelihood of skull fractures being found, and NAI as the underlying cause for the skull fracture.

\section{G334}

TAKING THE 'OUCH!' OUT OF EMERGENCY: USING ILLUSTRATIVE AND DIGITAL ARTS TO GUIDE, REASSURE AND ROLE MODEL BEHAVIOURS AT THE CHILDREN'S EMERGENCY DEPARTMENT, EVELINA LONDON CHILDREN'S HOSPITAL

1J Criddle, ${ }^{1} \mathrm{D}$ Hall, ${ }^{2} \mathrm{M}$ Jones, ${ }^{1} \mathrm{R}$ Mitchell. 'Children's Emergency Department, Evelina Children's Hospital, London, UK; ${ }^{2}$ Art in Site, London, UK

\subsection{6/archdischild-2018-rcpch.324}

Background Visiting the Emergency Department (ED) can be an emotive and stressful time for children and their families. A rebuild of The Childrens' Emergency Floor at Evelina London saw the space transformed to create an environment to help with the delivery of healthcare. Breaking away from traditional fantasy graphics, a new art-scheme aims to bring reassurance and stimulation to famililes and children, rolemodelling behaviours and acceptance.

Clinicians worked alongside 'Art In Site', using interactive storytelling to demystify the service. After conducting an environmental psychological study, the design team observed the workings of the department and held workshops with staff, psychologists, patients and families, forging strategic and design prototypes. The project was funded by Guy's and St Thomas's Charity and the art-scheme was installed in late 2016.

Outcomes The project incorporates the following features:

- 'Information Slices': strategically placed text, informing patients at points of need, in simple, jargon-free language.

- A 'gang' of illustrated characters feature in an interactive app, helping demystify the Emergency process through:

- Interactive animations explaining common procedures

- Stories depicting common causes of visits to ED

- Interactive X-ray visualisations of broken bones (and how they'll be fixed)

- Advice to teens on healthy-living, sexual and mental health

- Parental advice about accident prevention and managing illness at home

- Characters integrate with the environment, 'popping out' from doorways, across walls, creeping onto ceilings, and appearing on stickers given to children. They function as information-givers throughout the patient journey.

- 'Bodypaint': a digital interactive installation projected onto a waiting room wall. Dynamic colours and shapes move in response to body movements.

A detailed evaluation is underway but the new scheme has been transformative. The waiting room is calmer, and children and families have a better understanding of their journey through ED. Stress and anxiety have reduced with a subsequent improvement in efficiency.

Conclusions Characters in apps and digital art can role-model clinical scenarios for children. This increases efficiency by reducing anxiety and improving interactions between clinicians, children and their families. Modelling other scenarios, such as common accidents, has potential to educate children and families and may have longer-term public health benefits. 